\title{
A GRAPH-THEORETICAL GENERALIZATION OF A CANTOR THEOREM
}

\author{
SIEMION FAJTLOWICZ
}

\begin{abstract}
In 1962 Gleason and Dilworth found a poset-theoretical generalization of the Cantor Theorem concerning the cardinality of power sets. In the present paper we prove a graph-theoretical generalization of both theorems.
\end{abstract}

0 . Dilworth and Gleason have proved in [2] (see also [1, Theorem 4.6]) that no order preserving map from a subset of a partially ordered set $P$ into $I(P)$, the set of all ideals ${ }^{1}$ of $P$, ordered by inclusion, is onto. Their theorem was a generalization of the well-known Cantor Theorem concerning the cardinality of power sets.

Our main purpose is to generalize the former result as follows: if $P$ and $Q$ are two directed graphs and $Q$ is a reflexive nondiscrete graph with at least two elements, then no antihomomorphism from a subgraph of $P$ into $H(P, Q)$, is onto. Here $H(P, Q)$ denotes the graph of all homomorphisms from $P$ into $Q$ considered as a subgraph of $Q^{P}$, i.e. with the relation defined coordinatewise. The theorem of Dilworth and Gleason can now be obtained by taking $Q=\{0,1\}$ with $1 \geqslant 0$, in which case we have that if $P$ is a partially ordered set, then $I(P)$ is anti-isomorphic to $H(P, Q)$.

If $Q$ is a symmetric graph then our theorems yield corresponding theorems for homomorphisms. In general, Theorems $1-4$ imply similar results for homomorphisms rather than antihomomorphisms. We shall postpone proofs to a sequel of this paper in which we shall study properties, defined below, $\mathrm{C}$ and $\mathrm{C}^{*}$ with respect to homomorphisms of arbitrary relational structures.

1. By a relational structure we shall mean here a pair $A=\left(A,\left(R_{t}\right)_{t \in T}\right)$, where for every $t \in T, R_{t}=R_{t}(A)$ is a binary relation on the nonempty set $A$. If $A$ and $B$ are two relational systems of the same type, then the function $f$ : $A \rightarrow B$ is said to be a homomorphism if for every $t \in T$ and $(a, b) \in R_{t}(A)$ we have that $(f(a), f(b)) \in R_{t}(B)$. If $(a, b) \in R_{t}(A)$ implies that $(f(b), f(a))$ $\in R_{t}(B)$, then $f$ is said to be an antihomomorphism.

If $A$ and $B$ are two systems of the same type, then $H(A, B)$ denotes a relational system, the universe of which is the set of all homomorphisms from

Presented to the Society, August 25, 1976; received by the editors May 4, 1976.

AMS (MOS) subject classifications (1970). Primary 05C20; Secondary 02K99.

${ }^{1}$ I.e. subsets $I \subseteq P$ such that $y \leqslant x \in I$, then $y \in I$. 
$A$ into $B$ and the relations of which are defined coordinatewise. When we say that $B$ is a subsystem of $A$ we mean that $B \subseteq A$ and for every $t \in T$, we have that $R_{t}(B)=B^{2} \cap R_{t}(A)$. A system with one relation will be called a graph, $|A|$ denotes the cardinality of the set $A$. A relational structure is nontrivial if it has more than one element and the relational structure is nondiscrete if there are $t \in T$ and $x \neq y$ such that $(x, y) \in R_{t}$.

A relational structure $Q$ has property $\mathrm{C}$ if for every structure $P$ of the same type as $Q$ there are no antihomomorphisms from $P$ onto $H(P, Q)$. Moreover, $Q$ has property $\mathrm{C}^{*}$ if for every substructure $P_{0}$ of $P$ there are no antihomomorphisms from $P_{0}$ into $H(P, Q)$.

2.

THEOREM 1. Let $Q$ be a relational system in which each of the relations $R_{t}$ is reflexive. If $Q$ has exactly two elements then $Q$ has property $\mathrm{C}$.

Proof. Let $P$ be a relational structure of the same type as $Q$ and let $\varphi$ be an antihomomorphism from $P$ into $H(P, Q)$. Let $Q=\{0,1\}$, and let $\phi$ : $P \rightarrow Q$ be defined as follows: $\phi(x)=1$ if and only if for every $y \in P$ we have that $\varphi(x) y=1$ implies that $\varphi(y) y=0$. We shall show that $\phi$ is a homomorphism. Suppose that $(a, b) \in R_{t}(P)$. Because each of the relations $R_{t}(Q)$ is reflexive, we obviously can assume that $\phi(a) \neq \phi(b)$, say $\phi(a)=0$ and $\phi(b)=1$. Because $\phi(a)=0$, there is $y \in P$, such that $\varphi(a) y=1$ and $\varphi(y) y$ $=1$. Because $\varphi(y) y=1$ and $\phi(b)=1$ we have that $\varphi(b) y=0$. Since $\varphi$ is an antihomomorphism into $H(P, Q)$ and $(a, b) \in R_{t}$, we must have that for every $y \in P$ the pair $(\varphi(b) y, \varphi(a) y) \in R_{t}(Q)$. Thus $(0,1) \in R_{t}(Q)$, i.e. $\phi$ is a homomorphism. We shall show now that $\phi \notin \operatorname{Im} \varphi$. In fact, suppose that $\phi=\varphi(e)$ and $\phi(e)=1$. Then $\varphi(e) e=1$ and thus, by the definition of $\phi$, we must have that $\varphi(e) e=0$, which is a contradiction. Suppose that $\phi(e)=0$. Then there exists $y$ such that $\varphi(e) y=1$ and $\varphi(y) y=1$. But $\varphi(e) y=1$ means that $\phi(y)=1$, which together with $\varphi(y) y=1$ implies that $\varphi(y) y=0$, which is a contradiction. Thus Theorem 1 is proved.

THEOREM 2. Let $Q$ be a relational structure containing a nontrivial reflexive substructure $Q_{0}$ injective in the category of all structures of the given type. If $Q_{0}$ has property $\mathrm{C}$ then $Q$ has property $\mathrm{C}^{*}$.

Proof. Let $P$ be a structure of the same type as $Q, P_{0}$ a substructure of $P$ and suppose that $\varphi: P_{0} \rightarrow H(P, Q)$ is an antihomomorphism onto $H(P, Q)$. Let $P_{1}$ be the inverse image of $H\left(P, Q_{0}\right)$ (note that $\left|H\left(P, Q_{0}\right)\right|>1$, because $Q_{0}$ is nontrivial and reflexive). Then $\varphi_{1}=\varphi \mid P_{1}$ maps $P_{1}$ onto $H\left(P, Q_{0}\right)$. Let us put $\gamma(h)=h \mid P_{1}$ for $h \in H\left(P, Q_{0}\right)$. Since $Q_{0}$ is injective, $\gamma$ is onto; since $\gamma$ is a homomorphism, $\varphi_{2}=\gamma \circ \varphi_{1}$ is an antihomomorphism. Thus $\varphi_{2}$ is an antihomomorphism from $P_{1}$ onto $H\left(P_{1}, Q_{0}\right)$ which is a contradiction because $Q_{0}$ has property $\mathrm{C}$. Thus $Q$ has property $\mathrm{C}^{*}$.

THEOREM 3. Every nondiscrete reflexive graph has property $\mathrm{C}^{*}$. 
Proof. Every nondiscrete graph contains a two-element nondiscrete subgraph, and this, by Theorem 1, has property $\mathrm{C}$. On the other hand, it is easy to see that every two-element reflexive graph is injective in the category of all graphs. Thus Theorem 3 follows from Theorem 2 .

THEOREM 4. Every nontrivial reflexive graph $Q$ has property $\mathrm{C}$.

Proof. In view of Theorem 3, we can assume that $Q$ is discrete. Let $\alpha(P)$ denote the number of connected components of the graph $P$. If $Q$ is discrete so is $H(P, Q)$. Because every homomorphism into a discrete graph is constant on connected components and arbitrary otherwise we have

$$
\alpha(H(P, Q))=|H(P, Q)|=|Q|^{\alpha(P)} \geqslant 2^{\alpha(P)}>\alpha(P) .
$$

Thus there are no antihomomorphisms from $P$ onto $H(P, Q)$.

\section{REFERENCES}

1. P. M. Cohn, Universal algebra, Harper and Row, New York,1965. MR 31 \#224; 32, p. 1754.

2. R. P. Dilworth and A. M. Gleason, A generalized Cantor theorem, Proc. Amer. Math. Soc. 13 (1962), 704-705. MR 26 \#2365.

Department of Mathematics, University of Houston, Houston, TeXas 77004 